\title{
Analysis of analog and RF behaviors in junctionless double gate vertical MOSFET
}

\author{
K. E. Kaharudin, Z. A. F. M. Napiah, F. Salehuddin, A. S. M. Zain, Ameer F. Roslan
}

MiNE, Centre for Telecommunication Research and Innovation, Faculty of Electronics and Computer Engineering, Universiti Teknikal Malaysia Melaka (UTeM), Hang Tuah Jaya, Durian Tunggal, 76100 Melaka

\begin{tabular}{l} 
Article Info \\
\hline Article history: \\
Received Aug 10, 2019 \\
Revised Oct 22, 2019 \\
Accepted Dec 1, 2019 \\
\hline
\end{tabular}

Keywords:

Cut-off frequency

Drain conductance

Gain band-width product

Intrinsic gate delay

Transconductance

\begin{abstract}
The prime obstacle in continuing the transistor's scaling is to maintain ultra-shallow source/drain (S/D) junctions with high doping concentration gradient, which definitely demands an advanced and complicated S/D and channel engineering. Junctionless transistor configuration has been found to be an alternative device structure in which the junction and doping gradients could be totally eliminated, thus simplifying the fabrication process. In this paper, a process simulation has been performed to study the impact of junctionless configuration on the analog and RF behaviors of double-gate vertical MOSFET. The result proves that the performance of $n$-channel junctionless double-gate vertical MOSFET (n-JLDGVM) is slightly better than the junction double-gate vertical MOSFET (n-JDGVM). Junctionless device exhibits better analog behaviors as the transconductance (gm) is increased by approximately $4 \%$. In term of RF behaviors, the junctionless device exhibits $3.4 \%$ and $7 \%$ higher cut-off frequency (fT) and gain band-width product (GBW) respectively over the junction device.
\end{abstract}

This is an open access article under the CC BY-SA license.

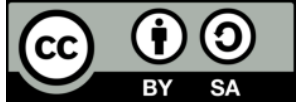

\section{Corresponding Author:}

F. Salehuddin,

Centre for Telecommunication Research and Innovation,

Faculty of Electronics and Computer Engineering,

Universiti TeknikalMalaysia Melaka (UTeM),

Hang Tuah Jaya, Durian Tunggal, 76100 Melaka.

Email: fauziyah@utem.edu.my

\section{INTRODUCTION}

The utilization of low power and high frequency based devices are very crucial for future electronic applications. Miniaturization in transistor's size with improvement in analog and RF performances is a vital goal of the microelectronics community. For realizing this goal, a number of device engineering methods such as source/drain engineering, multi-gate technology, dual material gate (DMG) technology, channel engineering, gate stack (GS) engineering and junctionless configuration have been carried out for decades [1-8]. According to International Technology Roadmap for Semiconductors 2013 (ITRS 2013) report [9], a precisely controlled process flow for the fabrication of transistors is becoming extremely complicated for deep sub-micron devices. As the transistor's size shrinks drastically towards sub-nanometer regime, the threshold voltage (VTH) would be rolled-off along with the decreasing channel length (Lch), which eventually deteriorating the overall performance due to short channel effects (SCE) [10]. The most difficult challenge in transistor's miniaturization is to form ultra-shallow source/drain (S/D) junctions with high doping gradient which requires advanced source/drain and channel engineering processes [11, 12]. For that reason, a lot of alternative device structures have emerged for realizing the Moore's law prediction without degrading the transistor performances. 
Recently, the junctionless transistor configuration has received a lot of attention in producing the ultra-small, low power and high frequency transistor due to its simplified fabrication process [6, 8, 13-16]. Instead of having the intricate source/drain and channel engineering processes, the junctions of the transistor can be completely removed by doping the source/drain and channel regions with similar polarity dopant, either n-type (for n-channel transistor) or p-type (for p-channel transistor) dopant. The junctionless-mode configuration could a void the adversity of forming the intricate junctions with high doping gradient, especially for short channel devices. The analog and RF behaviors in junctionless transistors are very important to be properly investigated, especially for system on chip (SoC) and system in package (SiP) applications [11, 17-19]. Thus, this paper emphasizes on the performance analysis of analog and RF behaviors of $\mathrm{n}$-channel junctionless double-gate vertical MOSFET (JLDGVM) and n-channel junction double-gate vertical MOSFET (JDGVM). The presented work in this paper is organized as follows: Section 2 briefly describes the device simulation of both JLDGVM and JDGVM and the process flow using Silvaco simulator. Section 3 discusses the comparative analysis of the analog and RF behaviors between n-JLDGVM and n-JDGVM in term of transconductance (gm), transconductance generation factor (TGF), output conductance (gd), early voltage (VEA), gate-to-source capacitance (Cgs), gate-to-drain (Cgd), parasitic capacitance (Cgg), intrinsic gate delay ( $\tau$ int), cut-off frequency (FT) and Gain Band-Width product (GBW). Finally, the conclusions and future work are presented in section 4.

\section{DEVICE SIMULATION}

The devices are designed based on high-k material (hafnium dioxide) as gate stack and extensive investigation is conducted by taking junctionless and junction configuration to verif $\mathrm{y}$ the effects on various analog and RF behaviors. The distinctive different between junctionless and junction configuration of double-gate vertical MOSFET is their opposite channel type as depicted in Figure 1. The channel region for n-JLDGVM is heavily doped with the similar polarity as source/drain doping in order to form $\mathrm{N}-\mathrm{N}+-\mathrm{N}$ type. In contrast, the channel region for n-JDGVM is doped with the opposite polarity as source/drain doping to form N-P-N type.

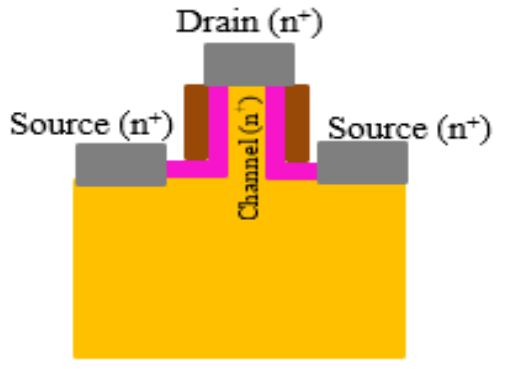

(a)

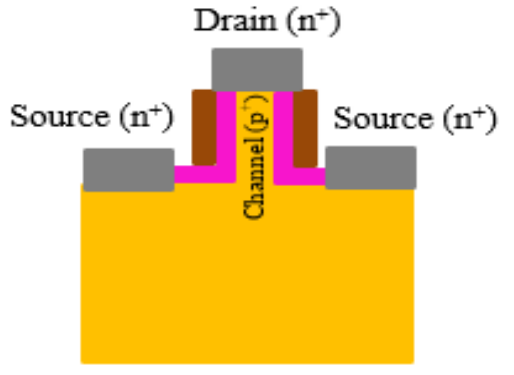

(b)

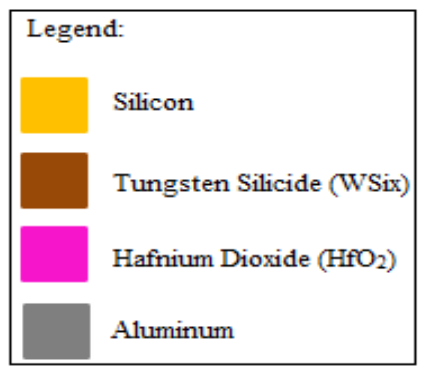

Figure 1. 2D structure of n-channel double-gate vertical MOSFETs, (a) Junctionless (n-JLDGVM), (b) Junction (n-JDGVM)

Extensive device simulation is performed via Silvaco Atlas for extracting the DC and AC characteristics of n-JLDGVM and JDGVM devices. The device simulation is conducted based on the current continuity and drift diffusion models in order to compute the current-voltage characteristics. Besides that, the mobility model is also included for considering the scattering processes such as lattice vibration (phonons), impurity ions, surfaces, and other material imperfections. The impact of these scattering process es would lead to low carrier mobility since they involve in both current continuity and drift-diffusion models [20]. For this case, the LombardiCVT model is opted to be employed to perform accurate simulation of non-planar MOSFET like JLDGVM and JDGVM devices.

\section{RESULTS AND DISCUSSION}

In this section, the simulation results and discussion are critically described for both n-JLDGVM and n-JDGVM devices. For the purpose of performance analysis of analog and RF behaviors in JLDGVM 
and JDGVM devices, the VTH values of both devices were fixed at $0.2 \mathrm{~V}$. The other DC characteristics are normalized to the fixed VTH value for unbiased performance evaluation. The DC analysis is an initial step for the further evaluation of analog and RF behaviors in both devices. The analog and RF behaviors for both devices are discussed in the following sub-sections.

\subsection{Analog behaviors}

Analog behaviors such as transconductance (gm), transconductance generation factor (TGF), drain conductance (gd) and early voltage (VEA) are presented and discussed here for analog performance point of view. The transconductance (gm) is an important figure of merit that implies how effective a transistor converts a voltage to a current. It is mainly employed for measuring the amplifier's gain. On the other hand, the transconductance generation factor (TGF) is also a significant figure of merit that implies how efficient a transistor converts DC power into AC frequency and gain. Figures 2 and 3 present the plot for gm and TGF respectively as a function of gate voltage (VG). The n-JLDGVM device demonstrates approximately $4 \%$ higher tranconductance (gm) than the JDGVM device. Below the gate voltage (VG) of $\sim 0.6 \mathrm{~V}$, the gm for both devices linearly increases as the $\mathrm{VG}$ increases. After $\mathrm{VG} \sim 0.6 \mathrm{~V}$, the gm for both devices begins to saturate until its reach maximum gate bias. It is observed that the maximum gm at VG=1 V for n-JLDGVM device is approximately $4 \%$ higher than the n-JDGVM device. Due to bulk phenomenon in junctionless configuration, the gm is slightly increased as it is approaching the maximum gate voltage. The improved gm value in junctionless configuration agreed with previous works conducted [16]. A higher gm of the n-JLDGVM device indicates that the conducting channel possesses higher transport efficiency which is suitable for analog based applications. In term of TGF, the n-JLDGVM device demonstrates approximately $20 \%$ higher TGF compared to JDGVM device. The slight improvement in TGF is in agreement with the results obtained by previous researchers [21,22]. The gm/ID ratio is mainly influenced by the pillar/body factor of the devices in weak inversion region. However, the gm/ID ratio for n-JDGVM is observed to be slightly decreased due to strong inversion in the channel region that causes a decrease in electron mobility at higher doping concentration.

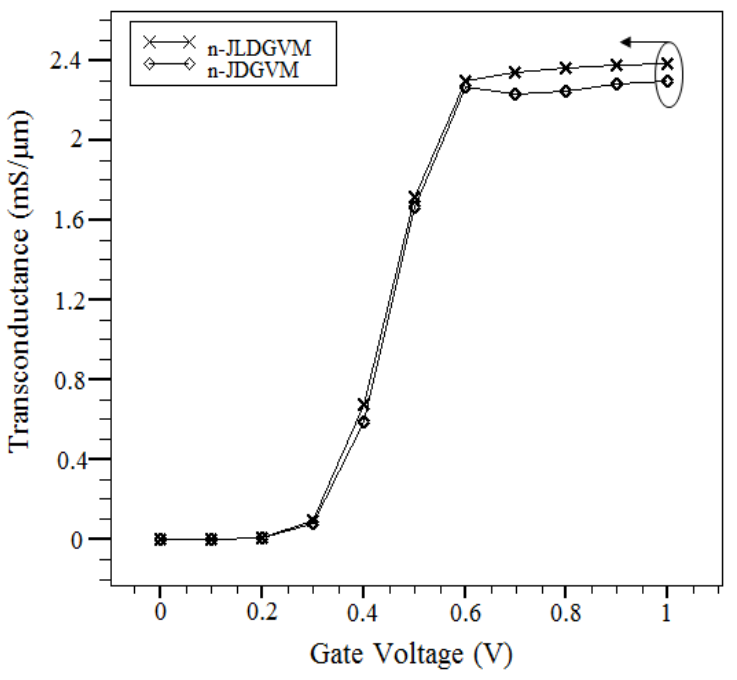

Figure 2. Transconductance (gm) as a function of gate voltage

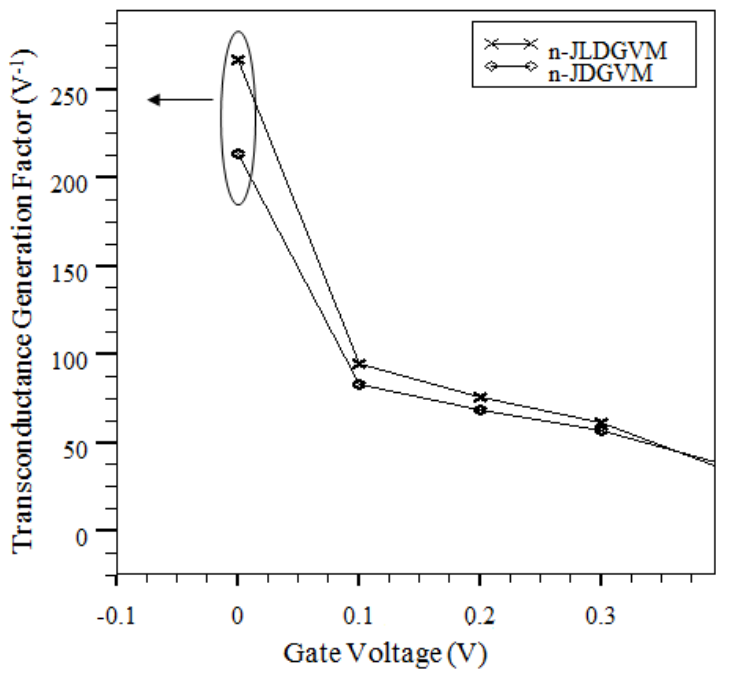

Figure 3. TGF as a function of gate voltage

The drain conductance $(\mathrm{gd})$ is also an important analog characteristic that decides a transistor's performance in analog based applications. The ultrathin pillar feature in both n-JLDGVM and n-JDGVM devices are very crucial in forming a fully depleted channel that could reduce the impact ionization effects. The impact ionization effects such as kink effect and parasitic bipolar action, normally experienced in most of bulk transistors would subsequently lead to a much higher gd. The value of gd is very crucia 1 in determining the drain current (ID) to drain conductance (gd) ratio, also called as early voltage (VEA). The VEA is not a constant value, but depends upon the channel length, inversion level and also the drain voltage (VD). Figures 4 and 5 show the plot of drain conductance (gd) and the early voltage (VEA) respectively at $\mathrm{VG}=0.5 \mathrm{~V}$ as a function of $\mathrm{VD}$. The $\mathrm{n}-\mathrm{JLDGVM}$ device carries higher drain current and thus results in higher drain conductance than the $n-J D G V M$ device. 


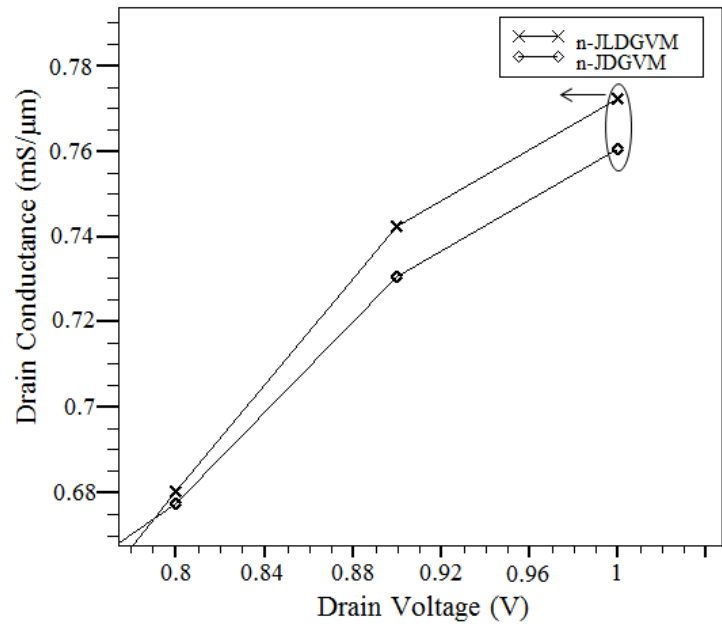

Figure 4. Drain conductance (gd) as a function of drain voltage

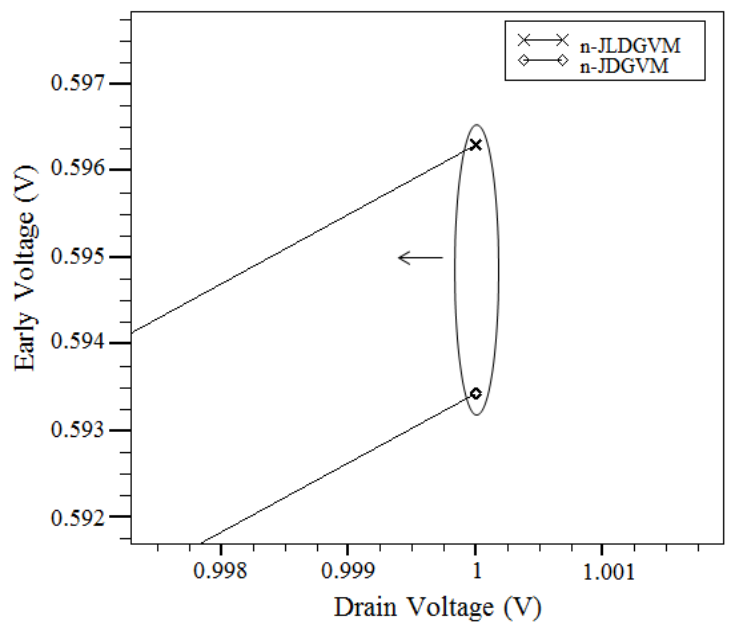

Figure 5. Early voltage (VEA) as a function of drain voltage

The n-JLDGVM device exhibits approximately $11 \%$ higher gd than n-JDGVM device. In most cases, the gd magnitude is strongly influenced by channel length modulation. In practical, CMOS analog circuits preferably need MOSFETs that exhibit low gd magnitude for attaining much higher gain. A slight improvement $(0.5 \%)$ in VEA can be observed in case of $n$-JLDGVM device as compared to n-JDGVM, mainly due to short channel effects (SCE) suppression. The side gates have better control over the charge carriers in the junctionless mode which slightly mitigating the SCE. As a result, the value of early voltage in n-JLDGVM device slightly increases and the dependence of the drain current on the drain voltage is minimized. Evidence of improved VEA for junctionless configuration is also reported by [11, 16]. The intrinsic gain (AV) is another important figure of merit for benchmarking the analog performance of operational transconductance amplifiers. The AV for the devices can be calculated as:

$$
A_{V}=\frac{g_{m}}{g_{d}}=\left(\frac{g_{m}}{I_{D}}\right) \times V_{E A}
$$

The extracted and computed values of gm, TGF, gd, VEA and AV for n-JLDGVM and n-JDGVM devices are summarized in Table 1. It is observed that the $n$-JLDGVM device exhibits marginally $2 \%$ higher AV than n-JDGVM device. Higher AV is pretty much desired in analog designs because it implies how efficient a transistor could amplify the input signal when it is biased to operate in both active and saturation region of operation.

Table 1. Analog performances for JLDGVM and JDGVM

\begin{tabular}{cccccc}
\hline Device & $\mathrm{g}_{\mathrm{m}}(\mathrm{mS} / \mu \mathrm{m})$ & $\mathrm{TGF}\left(\mathrm{V}^{-1}\right)$ & $\mathrm{g}_{\mathrm{d}}(\mathrm{mS} / \mu \mathrm{m})$ & $\mathrm{V}_{\text {EA }}(\mathrm{V})$ & $\mathrm{A}_{\mathrm{v}}(\mathrm{dB})$ \\
\hline JLDGVM & 2.39 & 267 & 0.772 & 0.596 & 9.8 \\
JDGVM & 2.30 & 214 & 0.761 & 0.593 & 9.6 \\
\hline
\end{tabular}

\subsection{RF behaviors}

Several crucial RF behaviors of the device are extracted and computed. Initially, the extractions of $\mathrm{Cgs}$ and $\mathrm{Cgd}$ are carried out through small signal analysis after post-processing DC analysis. The values of capacitance between source and drain regions are measured by supplying a single AC frequency (f) of $1 \mathrm{MHz}$ as the VG is swept from $0 \mathrm{~V}$ to $1 \mathrm{~V}$ with a step of $0.01 \mathrm{~V}$. Figures 6 and 7 depict the plot of intrinsic capacitances (Cgs and $\mathrm{Cgd}$ ) respectively as a function of $\mathrm{VG}$ at a fixed $\mathrm{VD}$ of $0.5 \mathrm{~V}$. Based on the combined plot, the Cgs for n-JLDGVM device is observed to be approximately $3 \%$ higher than the $n$-JDGVM device. Furthermore, both devices show an almost linear increase in Cgs value as the gate voltage is shifted towards its maximum value $(1 \mathrm{~V})$. The difference of $\mathrm{Cgs}$ for both devices is less prominent as it does not contribute significant changes in output current when the gate is turned of $\mathrm{f}$ and the time required for the output to turn off completely. On the other hand, the n-JLDGVM device exhibits approximately $3 \%$ lower Cgd compared to $\mathrm{n}-\mathrm{JDGVM}$ device. Both devices exhibit an almost constant Cgd value as the gate voltage is shifted towards its maximum value $(1 \mathrm{~V})$. The high $\mathrm{Cgd}$ is not desirable in RF and high frequency applications because it would delay the rise in drain current after the gate is turn on and the fall in drain current after the gate 
is turned off. As consequence, the switching speed is greatly reduced by the increasing Cgd. The Cgg, also known as the parasitic gate capacitance is an important characteristic for determining the intrinsic gate delay of a transistor. Figure 8 shows plot for the $\mathrm{Cgg}$ as a function of VG at a constant VD of $0.5 \mathrm{~V}$. The n-JLDGVM device demonstrates a slight decrease in Cgg over the n-JDGVM device. A larger Cgg definitely would result in higher gate delay.

The intrinsic gate delay is used to indicate the frequency limit of RF circuits. The intrinsic ga te delay of a transistor heavily depends on the parasitic gate capacitance $(\mathrm{Cgg})$. A higher intrinsic gate delay would affect the propagation of majority carriers (electrons), especially in high frequency applications. Figure 9

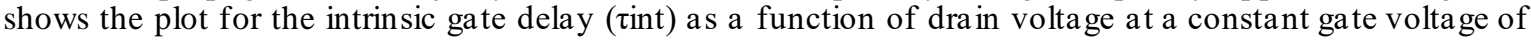
$0.5 \mathrm{~V}$. The $\mathrm{n}$-JLDGVM device exhibits approximately $3 \%$ lower $\tau$ int than $\mathrm{n}$-JDGVM device at maximum dra in voltage $(1 \mathrm{~V})$. Both devices demonstrate a significant increase in tint before the drain current saturates. After the drain current for both devices saturates, their corresponding iint begin to decline tremendously. Another transistor's characteristic that is crucial for RF and high frequency application is known as cut-off frequency (fT). The fT is a figure of merit of a transistor to reflect both frequency response and gain. The fT represents a unity gain frequency of a transistor amplifier that is majorly driven by input and output $\mathrm{RC}$ constants. The value of intrinsic capacitances is a dominant factor that drives the frequency response since the value of gm predominantly governs the voltage gain. Besides that, it is also important to consider the gain-band-width product (GBW) of both devices for the purpose of high frequency transient analysis.

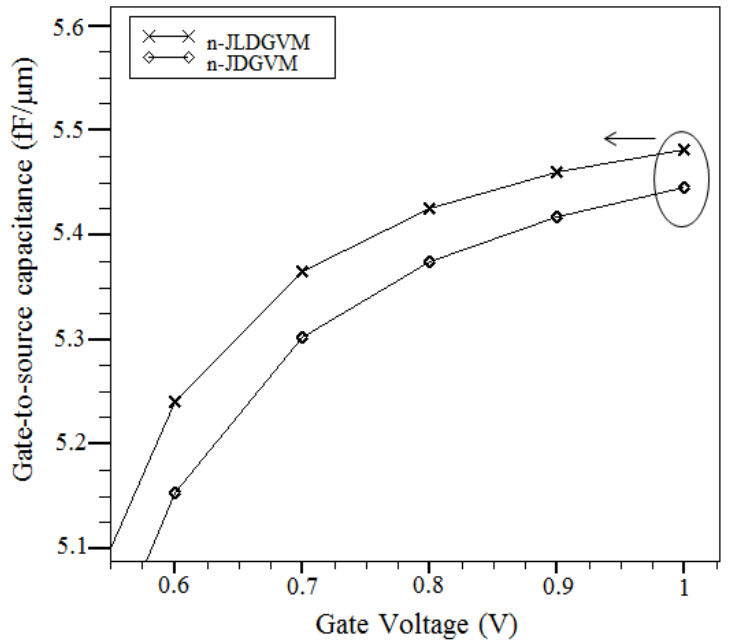

Figure 6. Cgs as a function of VG

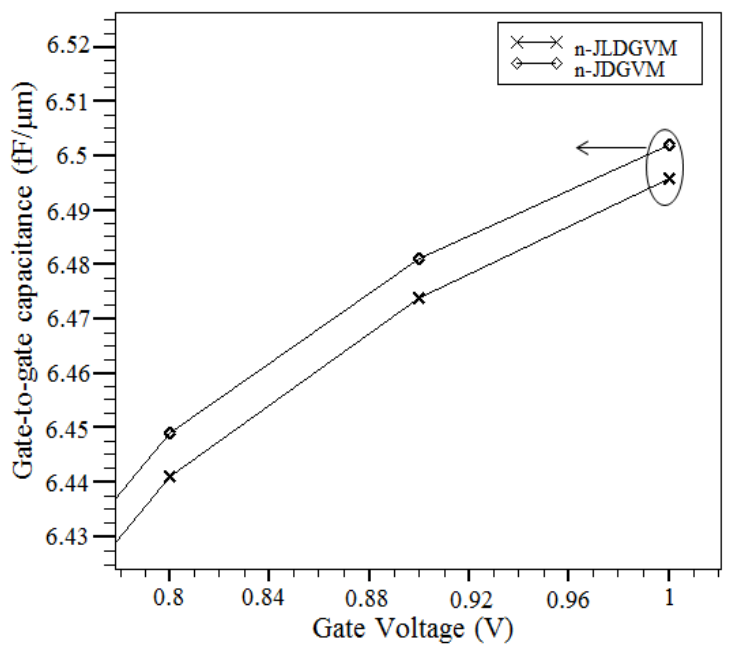

Figure 8. Cgg as a function of $\mathrm{VG}$

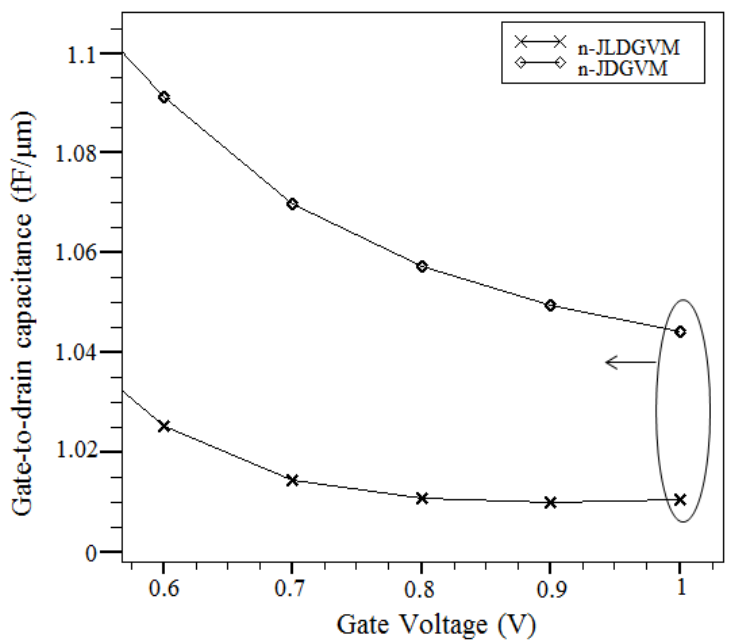

Figure 7. Cgd as a function of VG

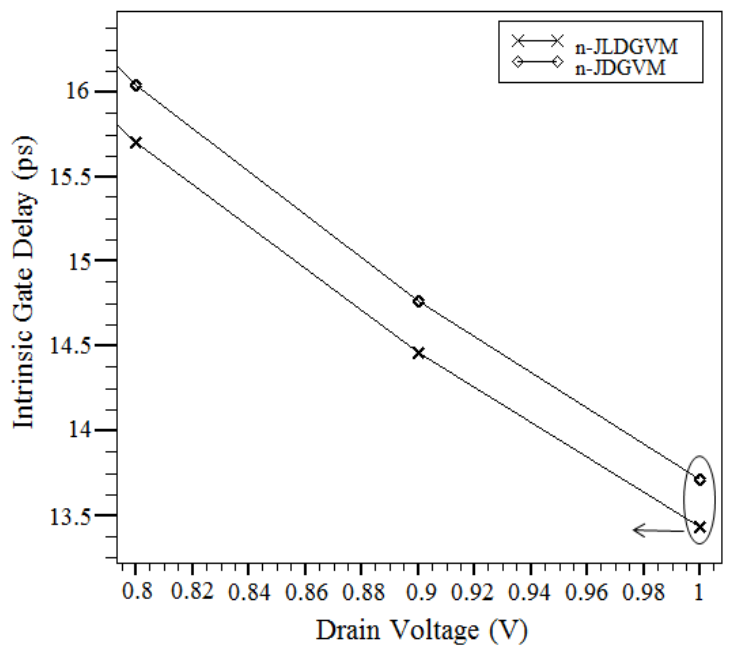

Figure 9. $\tau_{\text {int }}$ as a function of $V_{D}$

Figures 10 and 11 depict the plot of fT and GBW respectively for both devices as a function of gate voltage at a constant drain voltage of $0.5 \mathrm{~V}$. The $\mathrm{n}-\mathrm{JLDGVM}$ device exhibits approximately $3.4 \%$ higher fT 
compared to n-JDGVM device. The slight improvement in fT for n-JLDGVM device over the n-JDGVM device is due to junctionless configuration which alters the energy band structure of the conductive layer. As a result, the effective mass of electrons is slightly reduced, thus increasing its mobility. The variation of fT over the increasing gate voltage is mainly affected by the gm and the intrinsic capacitances. Hence, high gm and low intrinsic capacitances are significantly required in achieving better cut-off frequency (fT) for high speed RF applications. In term of GBW, the n-JLDGVM device shows $7 \%$ higher GBW than the n-JDGVM device. The minor improvement in GBW of n-JLDGVM device is mainly influenced by its higher gm and the lower Cgd than the n-JDGVM device. The high GBW is very desirable for circuit applications that require extremely high speed and low input bias current such as RF amplifiers [23]. The extracted and computed values of Cgs, Cgd, Cgg, tint, FT and GBW for n-JLDGVM and n-JDGVM devices are summarized in Table 2. Based on the obtained results, it can be concluded that the junctionless configuration does improve the overall device characteristics of double-gate vertical MOSFET, including the analog and RF behaviors. A slight improvement in ID, gm, TGF, gd, VEA, AV, Cgs, Cgd Cgg, tint, fT and GBW clearly indicate that the n-JLDGVM device can be a potential transistor configuration for future low power and high frequency analog and RF applications. However, the analog and RF behaviors for the n-JLDGVM device still could be further improved by optimizing the geometrical and process parameters. Thus, various optimization methods [24-30] could be applied to further enhance the DC and AC characteristics for analog and $\mathrm{RF}$ applications.

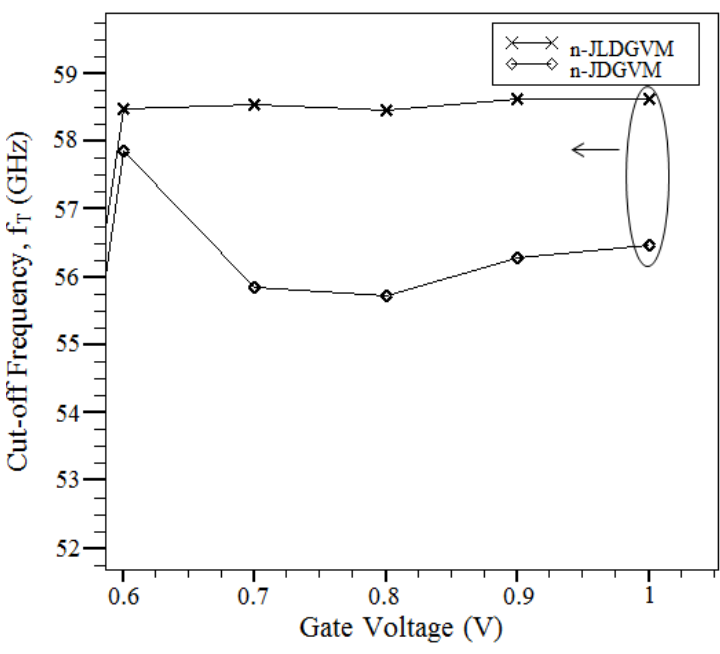

Figure 10. $\mathrm{f}_{\mathrm{T}}$ as a function of $\mathrm{V}_{\mathrm{G}}$

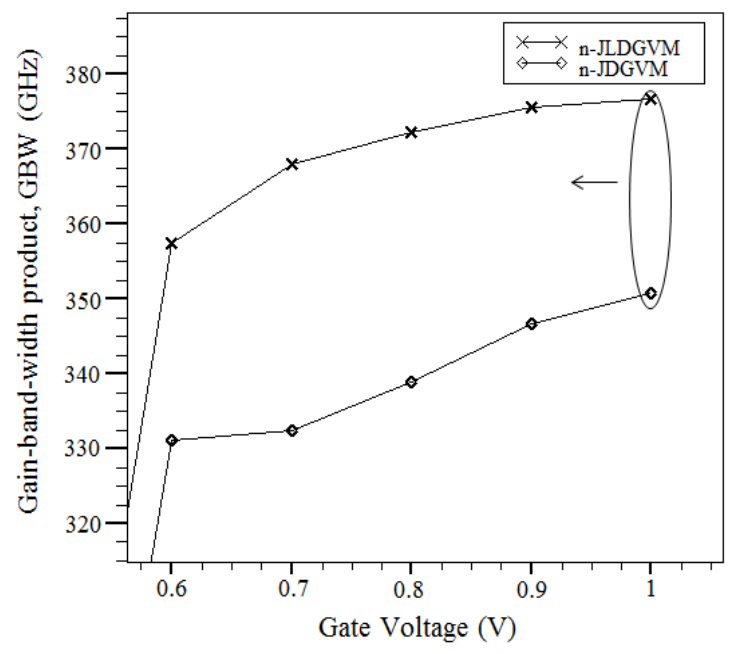

Figure 11. GBW as a function of VG

Table 2. RF performances for JLDGVM and JDGVM

\begin{tabular}{ccccccc}
\hline Device & $\mathrm{C}_{\mathrm{gs}}(\mathrm{fF} / \mu \mathrm{m})$ & $\mathrm{C}_{\mathrm{gd}}(\mathrm{fF} / \mu \mathrm{m})$ & $\mathrm{C}_{\mathrm{gg}}(\mathrm{fF} / \mu \mathrm{m})$ & $\tau_{\text {int }}(\mathrm{ps})$ & $\mathrm{f}_{\mathrm{T}}(\mathrm{GHz})$ & $\mathrm{GBW}(\mathrm{GHz})$ \\
\hline JLDGVM & 5.48 & 1.01 & 6.49 & 13.4 & 59 & 377 \\
JDGVM & 5.45 & 1.04 & 6.50 & 13.7 & 57 & 351 \\
\hline
\end{tabular}

\section{CONCLUSION}

In summary, the DC, analog and RF behaviors of $n$-channel junctionless double-gate vertical MOSFET (n-JLDGVM) are investigated and compared with the $n$-channel junction double-gate vertical MOSFET (n-JDGVM). The device performance between n-JLDGVM and n-JDGVM are compared in term of gm, TGF, gd, VEA, AV, Cgs, Cgd Cgg, int, fT and GBW. The results show that the junctionless device demonstrates slightly better analog behaviors over the junction device as the gm magnitude is increased by $4 \%$. In term of RF behaviors, the junctionless device exhibits $3.4 \%$ and $7 \%$ higher fT and GBW respectively over the junction device. These show that $n$-JLDGVM device can be a potential transistor configuration for future low power and high frequency a nalog and RF applications. Furthermore, both analog and RF behaviors of the n-JLDGVM device could be possibly improved by optimizing geometrical and process parameters through statistical methods or predictive analysis.

\section{ACKNOWLEDGEMENTS}

The authors would like to thank to the Ministry of Higher Education (MOHE) of Malaysia, Mybrain 15 and Centre for Telecommunication Research and Innovation (CeTRI), Faculty of Electronics and 
Computer Engineering (FKEKK), Universiti Teknikal Malaysia Melaka (UTeM) for sponsoring this research study under research grant PJP/2018/FKEKK(2B)/S01614).

\section{REFERENCES}

[1] J. Dubois, M. Bolt, P. Semiconductors, and H. Tuinhout, "Impact of source/drain implants on threshold voltage matching in deep sub-micron CMOS technologies," in European Solid-State Device Research Conference, pp. 115-118, 2002.

[2] S. K. Mohapatra, K. P. Pradhan, L. Artola, and P. K. Sahu, "Estimation of analog/RF figures-of-merit using device design engineering in gate stack double gate MOSFET," Materials Science in Semiconductor Processing, vol. 31, pp. 455-462, 2015.

[3] S. Archana, G. Vallathan, and M. A. Kumar, "Analytical modeling of dual material junctionless surrounding gate MOSFET," SSRG International Journal of Electronics and Communication Engineering, vol. 4, no. 3, pp. 40-43, 2017.

[4] Q. Xu et al., "Ion-implanted TiN metal gate with dual band-edge work function and excellent reliability for advanced CMOS device applications," in IEEE Transactions on Electron Devices, vol. 62, no. 12, pp. 4199-4205, Dec 2015.

[5] A. Kumar, M. M. Tripathi, and R. Chaujar, "Comprehensive analysis of sub-20 nm black phosphorus based junctionless-recessed channel MOSFET for analog/RF applications," Superlattices and Microstructures, vol. 116, no. February, pp. 171-180, 2018.

[6] N. F. Kosmani, F. A. Hamid, and M. A. Razali, "A comparison of performance between double-gate and gate-all-around nanowire MOSFET," Indonesian Journal of Electrical Engineering and Computer Science, vol. 13, no. 2, pp. 801-807, 2019.

[7] M. Khaouani and A. Guen-bouazza, "Impact of multiple channels on the characteristics of rectangular GAA MOSFET," International Journal of Electrical and Computer Engineering, vol. 7, no. 4, pp. 1899-1905, 2017.

[8] M. A. Riyadi, I. D. Sukawati, T. Prakoso, and Darjat, "Influence of gate material and process on junctionless FET subthreshold performance," International Journal of Electrical and Computer Engineering, vol. 6, no. 2, pp. 895-900, 2016.

[9] ITRS, "International Technology Roadmap Semiconductor," 2013.

[10] A. F. Roslan, F. Salehuddin, A. S. M. Zain, K. E. Kaharudin, I. Ahmad, and H. Hazura, "Comparative high-k material gate spacer impact in DG-FinFET parameter variations between two structures," Indonesian Journal of Electrical Engineering and Computer Science, vol. 14, no. 2, pp. 573-580, 2019.

[11] Y. Chen, M. Mohamed, M. Jo, U. Ravaioli, and R. Xu, "Junctionless MOSFETs with laterally graded-doping channel for analog/RF applications," Journal of Computational Electronics, vol. 12, no. 4, pp. 757-764, 2013.

[12] A. F. Roslan, F. Salehuddin, A. S. M. Zain, K. E. Kaharudin, H. Hazura, and A. R. Hanim, "30 nm DG-FinFET 3D construction impact towards short channel effects," Indonesian Journal of Electrical Engineering and Computer Science, vol. 12, no. 3, pp. 1358-1365, 2018.

[13] C. W. Lee, I. Ferain, A. Afzalian, R. Yan, N. D. Akhavan, P. Razavi, and J. P. Colinge, "Performance estimation of junctionless multigate transistors," Solid-State Electronics, vol. 54, no. 2, pp. 97-103, 2010.

[14] J. P. Colinge, C. W. Lee, I. Ferain, N. D. Akhavan, R. Yan, P. Razavi, R. Yu, A. N. Nazarov, and R. T. Doria, "Reduced electric field in junctionless transistors," Applied Physics Letters, vol. 96, no. 7, pp. 073510-1- 073510-3, 2010.

[15] J. P. Colinge, C. W. Lee, A. Afzalian, N. D. Akhavan, R. Yan, I. Ferain, P. Razavi, B. O’Neill, A. Blake, M. White, A. M. Kelleher, B. McCarthy, and R. Murphy, "Nanowire transistors without junctions," Nature Nanotechnology, vol. 5, no. 3, pp. 225-229, 2010 .

[16] R. T. Doria et al., "Junctionless multiple-gate transistors for analog applications," in IEEE Transactions on Electron Devices, vol. 58, no. 8, pp. 2511-2519, Aug 2011.

[17] P. K. Sahu, S. K. Mohapatra, and K. P. Pradhan, "Impact of downscaling on analog/RF performance of sub-100nm GS-DG MOSFET," Journal of Microelectronics, Electronic Components and Materials, vol. 44, no. 2, pp. 119-125, 2014.

[18] B. Lakshmi and R. Srinivasan, "Effect of process parameter variation on $\mathrm{ft}$ in coventional and junctionless gate-all-around devices," Journal of Engineering Science and Technology, vol. 10, no. 8, pp. 994-1008, 2015.

[19] F. Salehuddin, K. E. Kaharudin, A. S. M. Zain, A. K. M. Yamin, and I. Ahmad, "Analysis of process parameter effect on DIBL in n-channel MOSFET device using L27 orthogonal array," in International Conferences on Fundamental and Applied Sciences, AIP Conf. Proc., vol. 1621, no. 1, pp. 322-328, 2014.

[20] Silvaco, "Silvaco ATLAS manual Device Simulation Software," 2006.

[21] R. K. Baruah and R. P. Paily, "Analog performance of bulk planar junctionless transistor (BPJLT)," in Computing Communication \& Networking Technologies (ICCCNT), Third International Conference, pp. 1-4, 2012.

[22] J. P. Colinge, A. Kranti, R. Yan, I. Ferain, D. Akhavan, P. Razavi, C. W. Lee, R. Yu, and C. A. Colinge, "A Simulation Comparison between junctionless and inversion-mode MuGFETs.," ECS Transactions, vol. 35, no. 5, pp. 63-73, 2011.

[23] S. Singh and P. Kumar, "Transient analysis \& performance estimation of gate inside junctionless transistor (GI-JLT)," International Journal of Nuclear and Quantum Engineering, vol. 8, no. 10, pp. 1641-1645, 2014.

[24] K. E. Kaharudin, F. Salehuddin, A. S. M. Zain, and M. N. I. A. Aziz, "Comparison of Taguchi method and central composite design for optimizing process parameters in vertical double gate MOSFET," ARPN Journal of Engineering and Applied Sciences, vol. 12, no. 19, pp. 5578-5590, 2017.

[25] K. E. Kaharudin, F. Salehuddin, A. S. M. Zain, and M. N. I. A. Aziz, "Impact of different dose, energy and tilt angle in source/drain implantation for vertical double gate PMOS device," Journal of Telecommunication, Electronic and Computer Engineering, vol. 8, no. 5, pp. 23-28, 2016. 
[26] K. E. Kaharudin, A. H. Hamidon, and F. Salehuddin, "Implementation of Taguchi modeling for higher drive current (ION) in vertical DG-MOSFET device," Journal of Telecommunication, Electronic and Computer Engineering, vol. 6, no. 2, pp. 11-18, 2014.

[27] K. E. Kaharudin, F. Salehuddin, A. S. Zain, and M. N. I. Aziz, "Application of Taguchi-based grey fuzzy logic for simultaneous optimization in TiO2/WSix-based vertical double-gate MOSFET," Journal of Telecommunication, Electronic and Computer Engineering, vol. 9, no. 2-13, pp. 23-28, 2017.

[28] K. E. Kaharudin, F. Salehuddin, and A. S. M. Zain, "Optimization of electrical properties in $\mathrm{TiO}_{2} / \mathrm{WSix}^{-b a s e d}$ vertical DG-MOSFET using Taguchi-based GRA with ANN," Journal of Telecommunication, Electronic and Computer Engineering, vol. 10, no. 1, pp. 69-76, 2018.

[29] R. Norani, "A dynamic simulation on single gate junctionless field effect transistor based on genetic algorithm," Advances in Computer Science: an International Journal, vol. 3, no. 5, pp. 140-145, 2014.

[30] K. E. Kaharudin, F. Salehuddin, A. S. M. Zain, and M. N. I. A. Aziz, "Influence of halo and source/drain implant variations on the drive current in p-channel vertical double gate MOSFET," in Proceedings of Mechanical Engineering Research Day, pp. 33-34, 2016.

\section{BIOGRAPHIES OF AUTHORS}

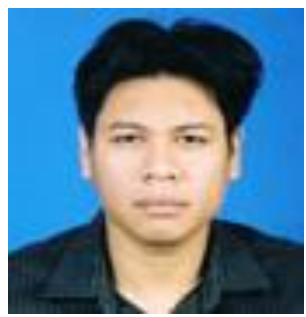

K. E. Kaharudin received Ph.D in Electronic Engineering and M. Eng degree in Computer Engineering from Technical University of Malaysia Melaka (UTeM), in 2017 and 2013 respectively. His Ph.D project focused on the process optimization of vertical double gate MOSFET. His research's interests include CMOS design, microelectronics, semiconductors, engineering optimization and artificial intelligence. Recently, his efforts emphasize on the simulation design of Junctionless MOSFET, silicon-on-insulator (SOI) MOSFET, high-k/metal-gate stack technology and design of experiment (DoE).

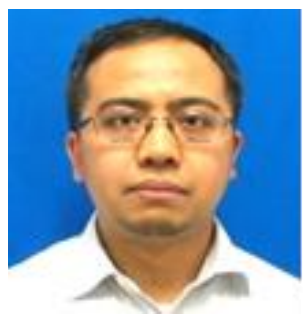

Z. A. F. M. Napiah received the B.Eng. degree in electrical engineering and the M.Eng. degree in Microelectronic from Universiti Teknologi Malaysia (UTM). He received the Ph.D. degree in Microelectronics from Kanawa University, Japan. He is currently a senior lecturer at Faculty of Electronic and Computer Engineering (FKEKK), UTeM. His research interest includes process and device simulation of MOSFET device, advanced CMOS design and CMOS based Photodetector and Photoreceiver

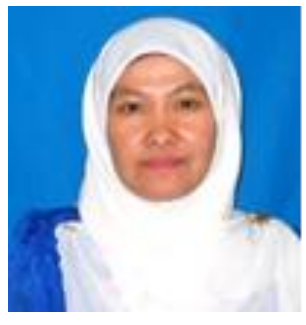

F. Salehuddin received the B.Eng. degree in electrical engineering (Communication) from Universiti Teknologi Mara (UiTM), Malaysia in 2001 and the M.Sc. degree in Electrical, Electronic and System Engineering from Universiti Kebangsaan Malaysia, in 2003. She received the Ph.D. degree in Microelectronics Engineering from Universiti Tenaga Nasional (UNITEN), Malaysia in 2012. She joined Universiti Teknikal Malaysia Melaka (UTeM) in December 2001 as a tutor and is currently a senior lecturer at Faculty of Electronic and Computer Engineering (FKEKK), UTeM. Her research interest includes process and device simulation of nanoscale MOSFETs device, advanced CMOS design, optimization approach (DOE) and process parameter variability.

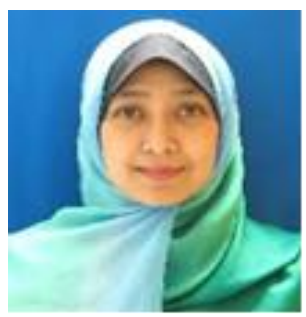

A. S. M. Zain received the B.Eng degree in electrical, Electronic and System engineering and M. Sc in Microelectronics from National University of Malaysia (UKM), Malaysia She received the Ph.D. degree in Nanoelectronics Engineering from University of Glasgow, UK in 2013. She is currently working as a senior lecturer at Faculty of Electronic and Computer Engineering (FKEKK), Universiti Teknikal Malaysia Melaka (UTeM). Her research interest includes Nanoscale Device Design and Simulation, Variability and Reliability of Emerging Technology Devices, IC Design for Biomedical Applications.

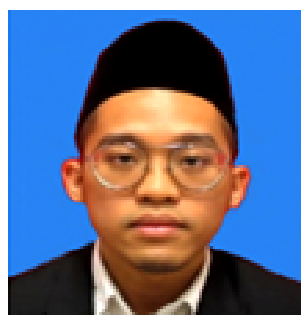

Ameer F. Roslan received by. Sc. Degree in Telecommunication Engineering from Technical University of Malaysia Melaka (UTeM), in 2017. He is currently pursuing his M.Sc degree in electronics and doing research on the DG-FinFET device at Centre for Telecommunication Research and Innovation, Faculty of Electronics and Computer Engineering, Technical University of Malaysia Melaka (UTeM). His research interest includes the DG-FinFET architecture and statistical 